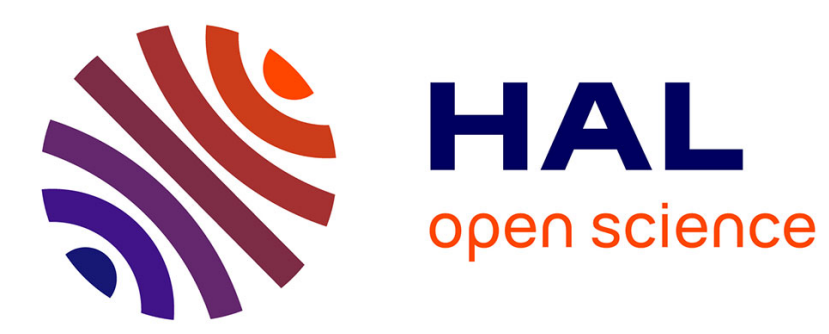

\title{
A Convergent Finite Volume Scheme for Two-Phase Flows in Porous Media with Discontinuous Capillary Pressure Field *
}

Clément Cancès, K Brenner, D. Hilhorst

\section{To cite this version:}

Clément Cancès, K Brenner, D. Hilhorst. A Convergent Finite Volume Scheme for Two-Phase Flows in Porous Media with Discontinuous Capillary Pressure Field *. FVCA 6 - International Symposium Finite Volumes for Complex Applications , 2011, Prague, Czech Republic. hal-01713549

\author{
HAL Id: hal-01713549 \\ https://hal.science/hal-01713549
}

Submitted on 20 Feb 2018

HAL is a multi-disciplinary open access archive for the deposit and dissemination of scientific research documents, whether they are published or not. The documents may come from teaching and research institutions in France or abroad, or from public or private research centers.
L'archive ouverte pluridisciplinaire HAL, est destinée au dépôt et à la diffusion de documents scientifiques de niveau recherche, publiés ou non, émanant des établissements d'enseignement et de recherche français ou étrangers, des laboratoires publics ou privés. 


\title{
A Convergent Finite Volume Scheme for Two-Phase Flows in Porous Media with Discontinuous Capillary Pressure Field *
}

\author{
K. Brenner, C. Cancès and D. Hilhorst
}

\begin{abstract}
We consider an immiscible incompressible two-phase flow in a porous medium composed of two different rocks. The flows of oil and water are governed by the Darcy-Muskat law and a capillary pressure law, where the capillary pressure field may be discontinuous at the interface between the rocks. Using the concept of multi-valued phase pressures, we introduce a notion of weak solution for the flow, and prove the convergence of a finite volume approximation towards a weak solution.
\end{abstract}

Key words: Finite volume, two phase flow, discontinuous capillary pressures MSC2010: 65M112, 76M12, 35K65, 76S05

\section{The Continuous Problem}

\subsection{Multivalued Phase Pressures}

Consider a heterogeneous porous medium, represented by a polygonal domain $\Omega \subset \mathbb{R}^{d}$, built of two homogeneous and isotropic subdomains, represented by polygonal domains $\Omega_{1}, \Omega_{2} \subset \mathbb{R}^{d}$. We assume that $\overline{\Omega_{1} \cup \Omega_{2}}=\bar{\Omega}$ and $\Omega_{1} \cap \Omega_{2}=\emptyset$, and we denote by $\Gamma$ the interface between the two rocks, i.e. $\bar{\Gamma}=\partial \Omega_{1} \cap \partial \Omega_{2}$. We consider two immiscible incompressible phases (e.g. water and oil), whose flows

Konstantin Brenner and Danielle Hilhorst

CNRS and Université Paris-Sud 11, 91405 Orsay Cedex, e-mail: konstantin.brenner@math.upsud.fr, danielle.hilhorst@math.u-psud.fr

Clément Cancès

LJLL, UPMC, 75252 Paris cedex 05, e-mail: cances@ann.jussieu.fr

* This work was supported by the GNR MoMaS (PACEN/CNRS, ANDRA, BRGM, CEA, EdF, IRSN), France. 
within $\Omega_{i}$ are described by the conservation of mass equations together with the Darcy-Muskat law:

$$
\begin{aligned}
\phi_{i} \partial_{t} s-\nabla \cdot\left(\eta_{o, i}(s)\left(\nabla p_{o}-\rho_{o} \mathbf{g}\right)\right) & =0, \\
-\phi_{i} \partial_{t} s-\nabla \cdot\left(\eta_{w, i}(s)\left(\nabla p_{w}-\rho_{w} \mathbf{g}\right)\right) & =0,
\end{aligned}
$$

where $s$ denotes the oil saturation of the fluid, $\phi_{i}>0$ the porosity of $\Omega_{i}$, the oil mobility $\eta_{o, i}$ is a Lipschitz continuous increasing function on $[0,1]$ satisfying $\eta_{o, i}(0)=0$, while the water mobility $\eta_{w, i}$ is Lipschitz continuous, decreasing on $[0,1]$ and such that $\eta_{w, i}(1)=0$. The density of the phase $\alpha(\alpha \in\{o, w\})$ is denoted by $\rho_{\alpha}$, and $\mathrm{g}$ is the gravity vector. Assume first that both phases coexist, i.e. $s \in(0,1)$, then each phase has its own pressure denoted by $p_{\alpha}$. Classically, they are supposed to be linked by the capillary pressure relation

$$
p_{o}-p_{w}=\pi_{i}(s)
$$

where the capillary pressure function $\pi_{i}$ is supposed to be increasing and to belong to $\mathscr{C}^{1}((0,1) ; \mathbb{R}) \cap L^{1}(0,1)$. Since the equation (1) degenerates, there is no control on the oil pressure $p_{o}$ on $\{s=0\} \cap \Omega_{i}$, excepted that, because of (3), one has $p_{o} \leq$ $p_{w}+\pi_{i}(0)$. Similarly, on $\{s=1\} \cap \Omega_{i}, p_{w} \leq p_{o}-\pi_{i}(1)$. In these cases, the pressure has to be considered as multivalued, i.e.

$$
s=0 \Leftrightarrow p_{o}=\left[-\infty, p_{w}+\pi_{i}(0)\right], \quad s=1 \Leftrightarrow p_{w}=\left[-\infty, p_{o}-\pi_{i}(1)\right] .
$$

We deduce from (4) that the capillary pressure function $\pi_{i}$ has to be extended into the monotone graph $\tilde{\pi}_{i}$, already introduced in $[3,5]$, defined by

$$
\tilde{\pi}_{i}(s)= \begin{cases}{\left[-\infty, \pi_{i}(0)\right]} & \text { if } s=0 \\ \pi_{i}(s) & \text { if } s \in(0,1) \\ {\left[\pi_{i}(1),+\infty\right]} & \text { if } s=1\end{cases}
$$

Note that there exists a continuous non-decreasing reciprocal function on $\mathbb{R}$, which we denote by $\tilde{\pi}_{i}^{-1}$.

At the interface $\Gamma$, we prescribe the continuity of the multivalued phase pressures

$$
p_{\alpha, 1} \cap p_{\alpha, 2} \neq \emptyset, \quad(\alpha \in\{o, w\})
$$

where $p_{\alpha, i}$ denote the trace of the pressure of the phase $\alpha$ on $\Gamma$ from $\Omega_{i}$. It is worth noticing that the condition (6) is equivalent to the continuity of the mobile phases prescribed in [8]. The volume conservation of each phase yields

$$
\sum_{i=1,2} \eta_{\alpha, i}\left(s_{i}\right)\left(\nabla p_{\alpha, i}-\rho_{\alpha} \mathbf{g}\right) \cdot \mathbf{n}_{i}=0
$$

where $\mathbf{n}_{i}$ denote the outward normal to $\partial \Omega_{i}$ w.r.t. $\Omega_{i}$. In order to close the problem, we prescribe the initial condition 


$$
s_{0} \in L^{\infty}(\Omega), \quad 0 \leq s_{0} \leq 1 \text { a.e. in } \Omega,
$$

and the null-flux boundary condition on $\partial \Omega_{i} \cap \partial \Omega$ :

$$
\eta_{\alpha, i}\left(s_{i}\right)\left(\nabla p_{\alpha, i}-\rho_{\alpha} \mathbf{g}\right) \cdot \mathbf{n}_{i}=0 .
$$

\subsection{Reformulation of the Problem}

We define the fractional flow function $f_{i}(s)=\frac{\eta_{o, i}(s)}{\eta_{o, i}(s)+\eta_{w, i}(s)}$. We introduce the Kirchhoff transform $\varphi_{i}(s)$ and the global pressure $P$ defined by

$$
\begin{aligned}
\varphi_{i}(s) & =\int_{0}^{s} f_{i}(a) \eta_{w, i}(a) \pi_{i}^{\prime}(a) d a, \\
P & =p_{w}+\lambda_{w, i}(\pi)=p_{o}+\lambda_{o, i}(\pi) \quad \text { for some } \pi \in \tilde{\pi}_{i}(s),
\end{aligned}
$$

where $\lambda_{w, i}(\pi)=\int_{0}^{\pi} f_{i} \circ \tilde{\pi}_{i}^{-1}(p) d p$ and $\lambda_{o, i}(\pi)=\lambda_{w, i}(\pi)-\pi$. Classical computations (see e.g. [7]) allow the rewrite the equations (1) as

$$
\phi_{i} \partial_{t} s-\nabla \cdot\left(\eta_{o, i}(s)\left(\nabla P-\rho_{o} \mathbf{g}\right)+\nabla \varphi_{i}(s)\right)=0,
$$

while the sum of the equations (1) and (2) yields

$$
-\nabla \cdot\left(M_{i}(s) \nabla P-\zeta_{i}(s) \mathbf{g}\right)=0,
$$

where $M_{i}(s)=\eta_{o, i}(s)+\eta_{w, i}(s) \geq \alpha_{M}>0$ and $\zeta_{i}(s)=\eta_{o, i}(s) \rho_{o}+\eta_{w, i}(s) \rho_{w}$. At the interface, the relations (6) have to be replaced (see [6]) by

$$
\exists \pi \in \tilde{\pi}_{1}\left(s_{1}\right) \cap \tilde{\pi}_{2}\left(s_{2}\right) \text { s.t. } P_{1}-\lambda_{w, 1}(\pi)=P_{2}-\lambda_{w, 2}(\pi) .
$$

We solve the problem on the domain $Q=\Omega \times(0, T)$ for some $T>0$, and we define $Q_{i}=\Omega_{i} \times(0, T)$.

Definition 1 (weak solution). A pair $(s, P)$ is said to be a weak solution of the problem if

1. $s \in L^{\infty}(Q)$ with $0 \leq s \leq 1$ a.e. in $Q, \varphi_{i}(s)$ and $P$ belong to $L^{2}\left((0, T) ; H^{1}\left(\Omega_{i}\right)\right)$;

2. there exists a measurable function $\pi$ mapping $\Gamma \times(0, T)$ to $\overline{\mathbb{R}}$ such that

$$
\pi \in \tilde{\pi}_{1}\left(s_{1}\right) \cap \tilde{\pi}_{2}\left(s_{2}\right) \text { and } P_{1}-\lambda_{w, 1}(\pi)=P_{2}-\lambda_{w, 2}(\pi) ;
$$

3. for all $\psi \in C_{c}^{\infty}(\bar{\Omega} \times[0, T))$, 


$$
\begin{gathered}
\iint_{Q} \phi s \partial_{t} \psi d x d t+\int_{\Omega} \phi s_{0} \psi(\cdot, 0) d x d t \\
-\sum_{i=1,2} \iint_{Q_{i}}\left(\eta_{o, i}(s)\left(\nabla P-\rho_{o} \mathbf{g}\right)+\nabla \varphi_{i}(s)\right) \cdot \nabla \psi d x d t=0, \\
\iint_{Q}\left(M_{i}(s) \nabla P-\zeta_{i}(s) \mathbf{g}\right) \cdot \nabla \psi d x d t=0 .
\end{gathered}
$$

Because of the choice of the boundary conditions, the global pressure $P$ is only defined up to a constant. In order to eliminate this degree of freedom, we prescribe that

$$
\int_{\Omega} P(x, t) d x=0, \quad \forall t>0 .
$$

The equation (13) can be reformulated as

$$
\nabla \cdot \mathbf{q}=0, \text { with } \mathbf{q}=-M_{i}(s) \nabla P+\zeta_{i}(s) \mathbf{g},
$$

while (12) can be rewritten under the form

$$
\phi_{i} \partial_{t} s+\nabla \cdot\left(\mathbf{q} f_{i}(s)+\gamma_{i}(s) \mathbf{g}-\nabla \varphi_{i}(s)\right)=0,
$$

with $\gamma_{i}(s)=\left(\rho_{o}-\rho_{w}\right) \eta_{w, i}(s) f_{i}(s)$.

\section{The Finite Volume Scheme}

Since nonlinear test functions are necessary for proving the convergence of the scheme, we must restrict our study to spatial discretizations satisfying an orthogonality condition, as developed in [9].

\section{Definition 2 (admissible discretization of $Q$ ).}

1. An admissible discretization of $\Omega$ is given by $\left(\mathscr{T}, \mathscr{E},\left(x_{K}\right)_{K \in \mathscr{T}}\right)$ where for all $K \in \mathscr{T}, K$ is an open polygonal subset of $\Omega$ such that $K \subset \Omega_{i}$ for some $i$. We define $\mathscr{T}_{i}=\left\{K \subset \Omega_{i}\right\}$, and we assume that $\bar{\Omega}_{i}=\overline{\bigcup_{K \in \mathscr{T}_{i}} K}$. For $K, L \in \mathscr{T}$ with $K \neq L$, then either the $(d-1)$-Lebesgue measure of $\bar{K} \cap \bar{L}$ is 0 , or there exists $\sigma \in \mathscr{E}_{K} \cap \mathscr{E}_{L}$ (denoted by $\sigma=K \mid L$ ) such that $\bar{\sigma}=\bar{K} \cap \bar{L}$. For all $K \in \mathscr{T}$, there exists $\mathscr{E}_{K} \subset \mathscr{E}$ such that $\partial K=\bigcup_{\sigma \in \mathscr{E}_{K}} \bar{\sigma}$. Moreover, $\mathscr{E}=\bigcup_{K \in \mathscr{T}} \mathscr{E}_{K}$. We define $\mathscr{E}_{\Gamma}=\{\sigma \in \mathscr{E}: \sigma \subset \Gamma\}, \mathscr{E}_{i}=\left\{\sigma \in \mathscr{E}: \sigma \subset \Omega_{i}\right\}$ and $\mathscr{E}_{\text {ext }}=\{\sigma \in \mathscr{E}: \sigma \subset \partial \Omega\}$, and set $\mathscr{E}_{K, \Gamma}=\mathscr{E}_{K} \cap \mathscr{E}_{\Gamma}, \mathscr{E}_{K, i}=\mathscr{E}_{K} \cap \mathscr{E}_{i}$. The family of points $\left(x_{K}\right)_{K \in \mathscr{T}}$ is such that $x_{K} \in K$ and if $\sigma=K \mid L$, the straight line $\left(x_{K} x_{L}\right)$ is orthogonal to $\sigma$. We denote by $d_{K, L}$ the distance between $x_{K}$ and $x_{L}$, and by $d_{K, \sigma}$ the distance between $x_{K}$ and $\sigma \in \mathscr{E}_{K}$. For all $K \in \mathscr{T}$ and $\sigma \in \mathscr{E}$ we denote by $m(K)$ and $m(\sigma)$ the corresponding Lebesgue measures.

2. Let $N$ be a positive integer, and $\delta t=T / N$; then a uniform discretization of $(0, T)$ is given by the family $\left(t^{n}\right)_{n \in\{0, \ldots, N\}}$, where $t^{n}=n \delta t$. 
3. A discretization $\mathscr{D}=\left(\mathscr{T}, \mathscr{E},\left(x_{K}\right)_{K \in \mathscr{T}},\left(t^{n}\right)_{n \in\{0, \ldots, N\}}\right)$ of $Q$ is said to be admissible if $\left(\mathscr{T}, \mathscr{E},\left(x_{K}\right)_{K \in \mathscr{T}}\right)$ is an admissible discretization of $\Omega$ and $\left(t^{n}\right)_{n}$ is a uniform discretization of $(0, T)$.

For a given admissible discretization $\mathscr{D}=\left(\mathscr{T}, \mathscr{E},\left(x_{K}\right)_{K \in \mathscr{T}},\left(t^{n}\right)_{n \in\{0, \ldots, N\}}\right)$ of $Q$, we define the quantities

$$
\operatorname{size}(\mathscr{T})=\max _{K \in \mathscr{T}} \operatorname{diam}(K), \quad \operatorname{reg}(\mathscr{T})=\max _{i=1,2} \max _{K \in \mathscr{T}}\left(\sum_{\sigma=K \mid L \in \mathscr{E}_{K, \mathrm{i}}} \frac{m(\sigma) d_{K, L}}{m(K)}\right),
$$

and

$$
\operatorname{size}(\mathscr{D})=\max (\operatorname{size}(\mathscr{T}), \delta t), \quad \operatorname{reg}(\mathscr{D})=\operatorname{reg}(\mathscr{T}) .
$$

Remark 1. The choice of uniform time steps is not necessary, and all the results presented here can be adapted to the case of nonuniform time steps.

For $K \in \mathscr{T}_{i}$, we define $g_{K}(s)=g_{i}(s)$ for all functions $g$ whose definition depends on the subdomain $\Omega_{i}$, as for example $\phi_{i}, \varphi_{i}, M_{i}, f_{i}, \ldots$.

We propose a fully implicit cell-centered finite volume scheme for the problem, whose unknowns at each time step are $\left(s_{K}^{n}, P_{K}^{n}\right)_{K \in \mathscr{T}}$ and an interface unknown $\left(\pi_{\sigma}^{n}\right)_{\sigma \in \mathscr{E}_{\Gamma}}$. For all $\sigma \in \mathscr{E}_{K, \Gamma}$, we define $s_{K, \sigma}^{n}=\tilde{\pi}_{K}^{-1}\left(\pi_{\sigma}^{n}\right)$, so that, if $\sigma=K \mid L$, one directly has that

$$
\pi_{\sigma}^{n} \in \tilde{\pi}_{K}\left(s_{K, \sigma}^{n}\right) \cap \tilde{\pi}_{L}\left(s_{L, \sigma}^{n}\right) .
$$

The total flux balance equation (18) is discretized by

$$
\sum_{\sigma \in \mathscr{E}_{K}} m(\sigma) Q_{K, \sigma}^{n}=0, \quad \forall n \in\{1, \ldots, N\}, \forall K \in \mathscr{T},
$$

with

$$
Q_{K, \sigma}^{n}= \begin{cases}\frac{M_{K, L}\left(s_{K}^{n}, s_{L}^{n}\right)}{d_{K}, L}\left(P_{K}^{n}-P_{L}^{n}\right)+\mathscr{R}\left(Z_{K, \sigma} ; s_{K}^{n}, s_{L}^{n}\right) & \text { if } \sigma=K \mid L \in \mathscr{E}_{K, i}, \\ \frac{M_{K}\left(s_{K}^{n}\right)}{d_{K, \sigma}}\left(P_{K}^{n}-P_{K, \sigma}^{n}\right)+\mathscr{R}\left(Z_{K, \sigma} ; s_{K}^{n}, s_{K, \sigma}^{n}\right) & \text { if } \sigma \in \mathscr{E}_{K, \Gamma}, \\ 0 & \text { if } \sigma \in \mathscr{E}_{K, \mathrm{ext}}\end{cases}
$$

where $M_{K, L}\left(s_{K}^{n}, s_{L}^{n}\right)=M_{L, K}\left(s_{L}^{n}, s_{K}^{n}\right)$ is an average of $M_{K}\left(s_{K}^{n}\right)$ and $M_{L}\left(s_{L}^{n}\right)$. For example, we can suppose, as in [10] that it is given by the harmonic mean

$$
M_{K, L}\left(s_{K}^{n}, s_{L}^{n}\right)=\frac{M_{K}\left(s_{K}^{n}\right) M_{K}\left(s_{L}^{n}\right) d_{K, L}}{d_{L, \sigma} M_{K}\left(s_{K}^{n}\right)+d_{K, \sigma} M_{K}\left(s_{L}^{n}\right)} .
$$

The function $Z_{K, \sigma}$ is defined by $Z_{K, \sigma}(s)=\zeta_{K}(s) \mathbf{g} \cdot \mathbf{n}_{K, \sigma}$, where $\mathbf{n}_{K, \sigma}$ denotes the outward normal to $\sigma$ with respect to $K$. For a function $f$, we denote by $\mathscr{R}(f ; a, b)$ the Riemann solver

$$
\mathscr{R}(f ; a, b)=\left\{\begin{array}{l}
\min _{c \in[a, b]} f(c) \text { if } a \leq b, \\
\max _{c \in[b, a]} f(c) \text { if } b \leq a .
\end{array}\right.
$$


The oil-flux balance equation (19) is discretized in the form

$$
\phi_{K} \frac{s_{K}^{n}-s_{K}^{n-1}}{\delta t} m(K)+\sum_{\sigma \in \mathscr{E}_{K}} m(\sigma) F_{K, \sigma}^{n}=0, \quad \forall n \in\{1, \ldots, N\}, \forall K \in \mathscr{T},
$$

with

$$
F_{K, \sigma}^{n}=\left\{\begin{array}{l}
Q_{K, \sigma}^{n} f_{K}\left(\bar{s}_{K, \sigma}^{n}\right)+\mathscr{R}\left(G_{K, \sigma} ; s_{K}^{n}, s_{L}^{n}\right)+\frac{\varphi_{K}\left(s_{K}^{n}\right)-\varphi_{K}\left(s_{L}^{n}\right)}{d_{K, L}} \text { if } \sigma=K \mid L \in \mathscr{E}_{K, i}, \\
Q_{K, \sigma}^{n} f_{K}\left(\bar{s}_{K, \sigma}^{n}\right)+\mathscr{R}\left(G_{K, \sigma} ; s_{K}^{n}, s_{K, \sigma}^{n}\right)+\frac{\varphi_{K}\left(s_{K}^{n}\right)-\varphi_{K}\left(s_{K, \sigma}^{n}\right)}{d_{K, \sigma}} \text { if } \sigma \in \mathscr{E}_{K, \Gamma}, \\
0 \text { if } \sigma \in \mathscr{E}_{K, \text { ext }},
\end{array}\right.
$$

where $G_{K, \sigma}(s)=\gamma_{K}(s) \mathbf{g} \cdot \mathbf{n}_{K, \sigma}$ and $\bar{s}_{K, \sigma}^{n}$ is the upstream value defined by

$$
\bar{s}_{K, \sigma}^{n}= \begin{cases}s_{K}^{n} & \text { if } Q_{K, \sigma}^{n} \geq 0 \\ s_{L}^{n} & \text { if } Q_{K, \sigma}^{n}<0 \text { and } \sigma=K \mid L \in \mathscr{E}_{K, i}, \\ s_{K, \sigma}^{n} & \text { if } Q_{K, \sigma}^{n}<0 \text { and } \sigma \in \mathscr{E}_{K, \Gamma}\end{cases}
$$

The interface values $\left(\pi_{\sigma}^{n}, P_{K, \sigma}^{n}, P_{L, \sigma}^{n}\right)$ for $\sigma=K \mid L \in \mathscr{E}_{\Gamma}$ are defined by the following nonlinear system:

$$
\begin{gathered}
P_{K, \sigma}^{n}-\lambda_{w, K}\left(\pi_{\sigma}^{n}\right)=P_{L, \sigma}^{n}-\lambda_{w, L}\left(\pi_{\sigma}^{n}\right) . \\
Q_{K, \sigma}^{n}+Q_{L, \sigma}^{n}=0 \\
F_{K, \sigma}^{n}+F_{L, \sigma}^{n}=0 .
\end{gathered}
$$

Note that since the equations (25) and (26) are linear with respect to $P_{K, \sigma}^{n}$ and $P_{L, \sigma}^{n}$, one can eliminate these interface values, only keeping $\pi_{\sigma}^{n}$. We impose the discrete counterpart of (17), that is

$$
\sum_{K \in \mathscr{T}} m(K) P_{K}^{n}=0, \quad \forall n \in\{1, \ldots, N\} .
$$

The discrete initial data is given by:

$$
s_{K}^{0}=\frac{1}{m(K)} \int_{K} s_{0}(x) d x, \quad \forall K \in \mathscr{T},
$$

so that $0 \leq s_{K}^{0} \leq 1$.

Proposition 1 (existence of a discrete solution). For all $n \in\{1, \ldots, N\}$, there exists $\left(\left(s_{K}^{n}\right)_{K \in \mathscr{T}},\left(P_{K}^{n}\right)_{K \in \mathscr{T}},\left(\pi_{\sigma}^{n}\right)_{\sigma \in \mathscr{E}}\right)$ satisfying the relations (20)-(28). Moreover,

$$
0 \leq s_{K}^{n} \leq 1, \quad \forall K \in \mathscr{T} .
$$

The proof of Proposition 1 will be given in the forthcoming paper [2].

For an admissible discretization $\mathscr{D}$ of $Q$, we denote by $s_{\mathscr{D}}$ and $P_{\mathscr{D}}$ the piecewise constant functions defined almost everywhere by 


$$
s_{\mathscr{D}}(x, t)=s_{K}^{n}, \quad P_{\mathscr{D}}(x, t)=P_{K}^{n} \quad \text { if }(x, t) \in K \times\left(t^{n-1}, t^{n}\right] .
$$

We consider now a sequence $\left(\mathscr{D}_{m}\right)_{m>0}$ of admissible discretizations of $Q$ in the sense of Definition 2 such that size $\left(\mathscr{D}_{m}\right)$ tends to 0 and reg $\left(\mathscr{D}_{m}\right)$ remains uniformly bounded as $m$ tends to $\infty$. We denote by $\left(s_{\mathscr{D}_{m}}, P_{\mathscr{D}_{m}}\right)_{m}$ a corresponding sequence of discrete solutions, whose existence has been stated in Proposition 1.

Theorem 1 (main result). There exists a weak solution $(s, P)$ in the sense of Definition 1 such that, up to a subsequence,

$$
\begin{gathered}
s_{\mathscr{D}_{m}} \rightarrow \text { s and a.e. in } Q \text { as } m \rightarrow \infty, \\
P_{\mathscr{D}_{m}} \rightarrow P \text { weakly in } L^{2}(Q) \text { as } m \rightarrow \infty .
\end{gathered}
$$

The proof of Theorem 1 that we will present in the forthcoming paper [2] is based on compactness arguments, using the material developed in $[9,10]$. The proof adapts the steps that are given in [6] for the continuous frame.

\section{Numerical Results}

We consider a model porous medium $\Omega=(0,1)^{2}$ composed of two layers $\Omega_{1}=$ $\{(x, y) \in \Omega \mid y<\Gamma(x)\}$ and $\Omega_{2}=\{(x, y) \in \Omega \mid y>\Gamma(x)\}$, which have different capillary pressure laws. The fluid densities are given by $\rho_{o}=0.81, \rho_{w}=1$, and $\mathbf{g}=-9.81 \mathbf{e}_{y}$. We suppose that the porosity is such that $\phi_{i}=1, i \in\{1,2\}$, and we define the oil and water mobilities by

$$
\eta_{o, i}(s)=0.5 s^{2} \text { and } \eta_{w, i}=(1-s)^{2}, i \in\{1,2\} .
$$

Moreover we suppose that the capillary pressure curves have the form

$$
\pi_{1}(s)=s \text { and } \pi_{2}(s)=0.5+s .
$$

and that the initial saturation is given by

$$
s_{0}(x)= \begin{cases}0.3 & \text { if } x \in \Omega_{1}, \\ 0 & \text { otherwise }\end{cases}
$$

The flow is driven by buoyancy, making the oil move along $\mathbf{e}_{y}$ until it reaches the interface $\Gamma$. For $t \leq 0.11$, oil can not access the domain $\Omega_{2}$, since the capillary pressure $\pi_{1}\left(s_{1}\right)$ is lower than the threshold value $\pi_{2}(0)=0.5$, which is called the entry pressure. Hence the saturation below the interface $s_{1}$ increases, as well as the capillary pressure $\pi_{1}\left(s_{1}\right)$. As soon as the capillary pressure $\pi_{1}\left(s_{1}\right)$ reaches the entry pressure $\pi_{2}(0)$, oil starts to penetrate in the domain $\Omega_{2}$. Nevertheless, as pointed out in $[1,4]$, a finite quantity of oil remains trapped under the rock discontinuity. This phenomenon is called oil trapping. 

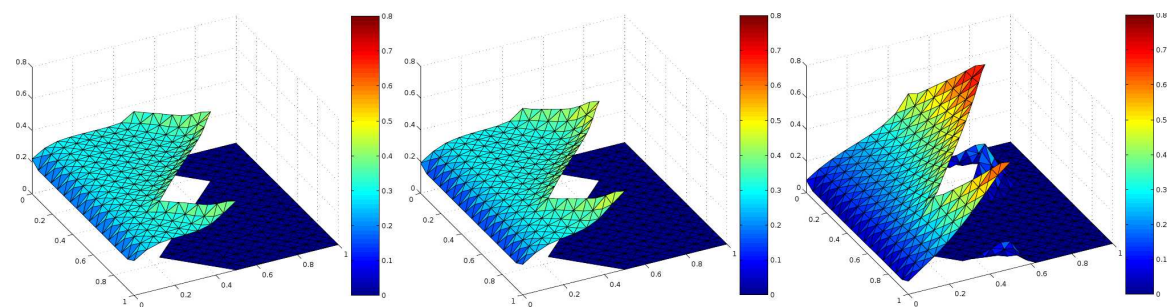

Fig. 1 Saturation for $t=0.06, t=0.11$ and $t=0.6$
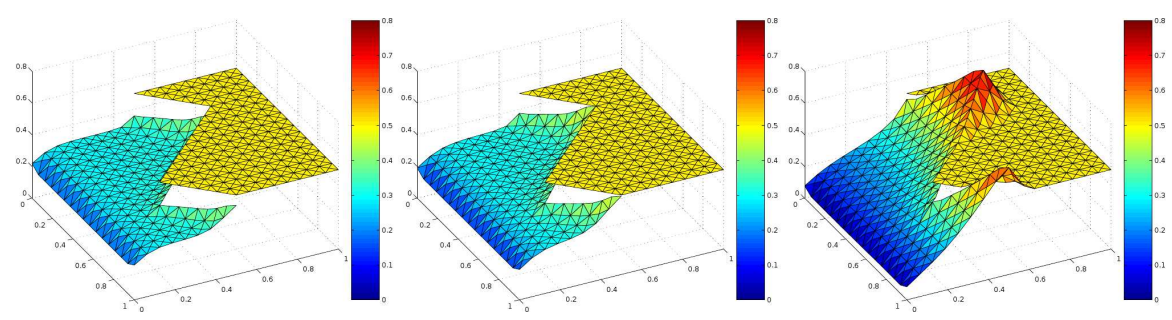

Fig. 2 Capillary pressure for $t=0.06, t=0.11$ and $t=0.6$

\section{References}

1. M. Bertsch, R. Dal Passo, and C. J. van Duijn. Analysis of oil trapping in porous media flow. SIAM J. Math. Anal., 35:245-267, 2003.

2. K. Brenner, C. Cancès, and D. Hilhorst. Convergence of a finite volume approximation of an immiscible two-phase flow in porous media with discontinuous capillary pressure field. In preparation.

3. F. Buzzi, M. Lenzinger, and B. Schweizer. Interface conditions for degenerate two-phase flow equations in one space dimension. Analysis, 29:299-316, 2009.

4. C. Cancès. Finite volume scheme for two-phase flow in heterogeneous porous media involving capillary pressure discontinuities. M2AN, 43:973-1001, 2009.

5. C. Cancès, T. Gallouët, and A. Porretta. Two-phase flows involving capillary barriers in heterogeneous porous media. Interfaces Free Bound., 11(2):239-258, 2009.

6. C. Cancès and M. Pierre. An existence result for multidimensional immiscible two-phase flows with discontinuous capillary pressure fields. HAL : hal-00518219, 2010.

7. G. Chavent and J. Jaffré. Mathematical Models and Finite Elements for Reservoir Simulation, volume 17. North-Holland, Amsterdam, stud. math. appl. edition, 1986.

8. G. Enchéry, R. Eymard, and A. Michel. Numerical approximation of a two-phase flow in a porous medium with discontinuous capillary forces. SIAM J. Numer. Anal., 43(6):2402-2422, 2006.

9. R. Eymard, T. Gallouët, and R. Herbin. Finite volume methods. Ciarlet, P. G. (ed.) et al., in Handbook of numerical analysis. North-Holland, Amsterdam, pp. 713-1020, 2000.

10. A. Michel. A finite volume scheme for two-phase immiscible flow in porous media. SIAM J. Numer. Anal., 41(4):1301-1317 (electronic), 2003. 